CLINICAL STUDY

\title{
IGF receptor gene variants in normal adolescents: effect on stature
}

\author{
Alvina R Kansra, Lawrence M Dolan ${ }^{1}$, Lisa J Martin ${ }^{1}$, Ranjan Deka ${ }^{2}$ and Steven D Chernausek ${ }^{3}$ \\ Department of Pediatrics, Medical College of Wisconsin, Milwaukee, Wisconsin 53226, USA, ${ }^{1}$ Department of Pediatrics, Cincinnati Children's Hospital \\ Medical Center, Cincinnati, Ohio 45229, USA, ${ }^{2}$ Department of Environmental Health, University of Cincinnati College of Medicine, Cincinnati, \\ Ohio 45269, USA and ${ }^{3}$ Department of Pediatrics, University of Oklahoma Health Sciences Center, 1200 North Phillips Avenue, Suite 4500, \\ Oklahoma City, Oklahoma 73104-4600, USA
}

(Correspondence should be addressed to S D Chernausek; Email: steven-chernausek@ouhsc.edu)

\begin{abstract}
Objective: IGF1 is essential for human growth and mediates its effects through the type 1 IGF receptor (IGF1R). Our objective was to determine the frequency of certain previously reported IGF1R gene variants in the normal population and their effect on stature.

Design: A cross-sectional study was conducted in a population of 2500 children enrolled in public school grades 5 through 12 for whom DNA and anthropometric data were available. Subjects were genotyped at five previously reported loci that affect receptor abundance or function.

Methods: The frequency of the following IGF1R variants Arg108Gln, Lys115Asn, Arg59stop, Arg481Gln, and Arg605His was measured by a PCR-based assay. Circulating concentrations of IGF1 or IGF binding protein-3 (IGFBP3) were measured by ELISA in those affected and matched controls. Results: A scan of 1300 subjects detected none with Arg108Gln, Lys115Asn, or Arg59stop mutations. In contrast, nucleotide changes leading to heterozygosity at codon 605 were identified in nine of 2500 subjects and six of 1800 subjects at codon 481 . These individuals were, on average, $4 \mathrm{~cm}$ shorter than the others. There were no differences in circulating concentrations of IGF1 or IGFBP3 between those with the gene variants and controls matched for sex, ethnicity, age, and BMI.

Conclusion: Rare IGF1R variants exerting a moderate effect on stature are present in the general population, supporting the importance of IGF1R function in growth control and indicating that variation in height within healthy individuals may be explained, in some cases, by larger effects of a small subset of gene variants.
\end{abstract}

European Journal of Endocrinology 167 777-781

\section{Introduction}

Approximately $80 \%$ of the variation in human stature is explained by heritable factors (1). Genome-wide association studies have identified more than 180 common genetic variants that influence stature within a general population, but in aggregate, these account for only 10\% of the variation in human height (2). Even though these common variants are related to stature across a wide range of height, there is also evidence that rare genetic variants with larger effects on stature are involved (3).

The type 1 insulin-like growth factor receptor gene (IGF1R) represents a locus where rare genetic variants might exert relatively large effects on stature. Normal abundance and function of the IGF1R is critical for normal growth as evidenced by gene deletion studies in mice (4) and reports of humans with variation in IGF1R number and sequence $(5,6,7,8,9,10,11,12,13,14$, $15,16,17)$. Homozygous $\operatorname{Igf} 1 r$ null mice are very small at birth and do not survive, and all affected humans described to date have genetic lesions compatible with partial IGF1R function. These findings imply that the complete lack of IGF1R signaling is incompatible with life and that graded reductions in IGF1R function will significantly attenuate growth.

We previously described two children with severe growth failure due to IGF1R mutations (10). In one of the cases reported, the child was a compound heterozygote for IGF1R mutations that reduced IGF1 binding and signaling. The below-average stature of her parents (height SDS - mother - 1.6, father -2.8), each of whom carried one mutant allele, led us to postulate that similar variations in IGF1R would be found to exert moderate effects on growth within the normal population. We tested this by assessing the frequency of five specific IGF1R variants in a school-based cohort of adolescents and determining the effect of these variants on stature. 


\section{Materials and methods}

\section{Study population}

Serum, DNA, and anthropometric data were obtained from individuals who participated in a school-based study that began in 2002 and was designed to assess risk, prevalence, and features of metabolic disease in children (18). In total 2501 of the 4273 students in grades 5 through 12 (ages 9.6-20.4 years) from a suburban school system participated in that study. Of those, $1263(50 \%)$ were non-Hispanic White and 1117 (45\%) African-American. The rest were Hispanic, Asian, West Indian, or multi-racial. Inclusion and exclusion criteria required each subject to have no known chronic disease and be taking no medication(s) known to affect carbohydrate metabolism. Pregnant females were excluded. The protocol was reviewed and approved by the Institutional Review Board at Cincinnati Children's Hospital Medical Center with written informed consent obtained from the parent/legal guardian and assent obtained from the subject.

\section{Study protocol}

Research subjects were evaluated annually. After a 10-h overnight fast, the following procedures were performed at the student's school: i) confirmation of the length of the fast and completion of the medical history; ii) height, weight, and documentation of the stage of axillary hair; and iii) venipuncture. Parents and students completed a personal medical history for each participant documenting chronic disease, medication use, and family history of diabetes. Blood samples were maintained on wet ice, processed within $3 \mathrm{~h}$ of venipuncture, and stored at $-20^{\circ} \mathrm{C}$.

Height and weight were measured using standard procedures and equipment as described previously (19). Pubertal status (prepubertal, pubertal and postpubertal) was assigned using criteria as described previously (18). For females, prepubertal status was defined as estradiol $<11 \mathrm{pg} / \mathrm{ml}$, pubertal was defined as estradiol $\geq 11 \mathrm{pg} / \mathrm{ml}$ without menarche, or duration of menarche $<2$ years, and postpubertal status was defined as the presence of menses for $>2$ years. In males, prepubertal status was defined as free testosterone $<1.0 \mathrm{pg} / \mathrm{ml}$, pubertal was defined as free testosterone $\geq 1.0 \mathrm{pg} / \mathrm{ml}$, and axillary hair equal to stage III, and postpubertal status was defined as free testosterone $\geq 1.0 \mathrm{pg} / \mathrm{ml}$ and axillary hair stage III. These cutoffs were derived from an analysis of subjects who had pubertal staging by standard physical examination and their discrimination validated by operator characteristic curve analysis (18). It was anticipated that those classified as postpubertal were at final or near-final height.

\section{Genotyping}

Five genetic variations in the IGF1R postulated to reduce IGF signaling were selected for examination. Published data indicated reduced IGF1R function or abundance in the case of 4: Arg108Gln - exon 2 (10), Lys115Asn - exon 2 (10), Arg59stop - exon 2 (10), and Arg481Gln - exon 7 (7). The fifth (Arg605His, exon 8) was selected because it was classified as "probably damaging' in the SNP database managed by the National Institutes of Environmental Sciences Environmental Genome Project (NIEHS SNPs Program, http:// egp.gs.washington.edu). Though other IGF1R mutations (Arg709Gln (9) and Glu1050Lys (8)) had been reported to cause short stature in humans at the time we initiated the study, we were unable to assess the frequency of these due to technical barriers with probe preparation.

A PCR-based assay with laser scanning technology to excite fluorescent dyes present in the custom-designed TaqMan probes (Applied Biosystems, 850 Lincoln Center Drive Foster City, CA 94404, USA) was used. Each primer pair consisted of two fluorogenic probes, labeled with two spectrally distinct dyes used to discriminate between alleles. Positive controls from individuals known to harbor the exon 2 variants were included in the assays. DNA sequencing was used to verify the detection of exon 7 and 8 variants.

\section{Hormone assays}

IGF 1 and IGF binding protein-3 (IGFBP3) were measured by ELISA using assay kits from Diagnostic Systems Laboratories (Webster, TX, USA) following the manufacturer's instructions. Intra- and interassay coefficients of variation were $<7 \%$. Free testosterone and estradiol were measured by RIA and competitive protein binding assay respectively as previously reported (20).

\section{Statistical analysis}

Data were examined for normality before analysis. All data were normally distributed, so no transformations were required. Because puberty is associated with accelerated growth velocity, we restricted the initial analysis to postpubertal measures of stature.

As these students participated in up to five annual study visits, we used mixed models analysis (Proc MIXED, SAS (version 9.1), Cary, NC, USA). Inclusion criteria for the analysis were either African-American or White self-reported race (due to the small number of other racial/ethnic groups) and postpubertal height data. The mixed models analysis accounts for both the intra- and the interindividual variability present in these data, resulting in larger, and more valid, estimates of standard error (21). Next, we evaluated the covariance structure of the data. Variance components, 
unstructured, and compound symmetry were examined. Compound symmetry fits the data best, so all models use compound symmetry for the covariance structure.

Because we expected the IGF1R mutations to be rare, we made an a priori decision to combine those harboring any of the mutations for analysis. Individuals were scored on the presence or absence of one or more of the IGF1R variants. Age, race, and sex were included as covariates. We considered significant effects to be present if $P \leq 0.05$.

\section{Results}

\section{Frequency of genetic variants}

A preliminary scan of 1300 subjects who were unrelated but otherwise randomly selected from the 2500 detected none with Arg108Gln, Lys115Asn, or Arg59stop mutations. Given the low frequency, no further testing for these mutations was conducted. In contrast, nucleotide changes leading to heterozygosity at codon 605 (rs45553041) were identified in nine of 2500 subjects, and six of 1800 were heterozygous at IGF1R residue 481 (codon 511, rs33958176). The frequencies of rs33958176 are consistent with those reported in the NCBI SNP database $(\mathrm{MAF}=0.001)$ (http://www.ncbi.nlm.nih.gov/snp) and the NHLBI exome database $(\mathrm{MAF}=0.0013) \quad$ (http://evs.gs. washington.edu/EVS/). SNP rs45553041 does not have a frequency listed in the NCBI SNP database and is not reported in the NHLBI exome database. Demographic, auxological, and hormonal data on those harboring the variants are displayed in Table 1 and shown in comparison with the 1800 controls that were postpubertal. Although there were apparent differences in sex and race distribution between variants and controls, these factors were accounted for in subsequent analyses. Circulating concentrations of testosterone appeared to be increased in those harboring IGF $1 R$ variants relative to controls, but the number of male subjects was small $(n=11)$ and could be due to other factors. Thus, the finding is of uncertain significance at this time. Resource constraints limited the analysis to only 1800 samples for the codon 511 variant; the limitation was arbitrary and there was no difference in gender, proportion of African-American and White participants, or heights from the 2500 subject group.

\section{Effect on stature}

After adjusting for age, sex, and race, postpubertal height was reduced in those subjects with the IGF $1 R$ variants by $4 \pm 2 \mathrm{~cm}(P=0.03)$ in mixed models. Because there were up to 4 yearly measures available for each subject, regression analyses were performed by study year, using mixed models accounting for repeated measures. Regression analysis provided similar effect sizes in all 4 years of data $(3.5-5.5 \mathrm{~cm}$ reduced height in those with a variant). In fact, there was no change in heights in the postpubertal subjects across those 4 years, irrespective of whether they harbored the variant or not, indicating that the variants reduced final height. Further, analysis of height for age $z$-scores resulted in similar effects (data not shown). None of the individuals harboring IGF1R variants enrolled before the onset of puberty. Therefore, the effect on prepubertal growth could not be assessed.

\section{Effect on circulating concentrations of IGF1 and IGFBP 3}

The reduction in stature associated with IGF $1 R$ variants suggested that these individuals were relatively IGF resistant. Previous reports indicate that IGF1 and IGFBP 3 levels are increased in some individuals with IGF resistance due to $\operatorname{IGF} 1 R$ mutations $(7,8,10,11)$. Therefore, we sought additional evidence for IGF resistance by comparing circulating concentrations of IGF 1 and IGFBP 3 in the IGF $1 R$ variant subjects with controls (Table 2). Each individual harboring an IGF1R variant was matched by age, sex, BMI, and race with three individuals from the study population who had none of the tested variants. All were postpubertal at the time of assessment. There were no differences between the variants and controls in the tabulated measures.

\section{Discussion}

Previous reports examining IGF $1 R$ variations in humans have either identified a pathogenic mutation in an affected individual with support of functional and

Table 1 Auxological and hormonal features of humans with IGF1R variants and controls.

\begin{tabular}{|c|c|c|c|}
\hline Feature & Arg481GIn $(n=6)$ & Arg605His $(n=9)$ & $\begin{array}{c}\text { Postpubertal } \\
\text { controls }(n=1800)\end{array}$ \\
\hline Sex, males (\%) & 66.7 & 62.5 & 43.2 \\
\hline Ethnicity: white \% & 33.3 & 11.1 & 45.7 \\
\hline Height percentile $^{a} \pm$ S.D. & $26.68 \pm 20.37$ & $15.99 \pm 26.04$ & $39.14 \pm 29.13$ \\
\hline Weight percentile $\mathrm{a}^{\mathrm{a}} \pm$ S.D. & $60.61 \pm 31.76$ & $63.69 \pm 31.50$ & $71.18 \pm 26.00$ \\
\hline Free testosterone, males $(\mathrm{pg} / \mathrm{ml})$ & $25.5 \pm 5.31$ & $18.92 \pm 9.90$ & $10.80 \pm 8.53$ \\
\hline Estradiol, females (pg/ml) & $54.54 \pm 37.09$ & $54.34 \pm 54.72$ & $45.80 \pm 46.76$ \\
\hline
\end{tabular}

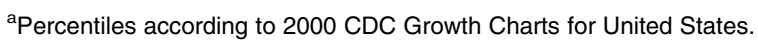


Table 2 IGF axis measures in IGF1R variants.

\begin{tabular}{lcc}
\hline Feature & $\begin{array}{c}\text { Variant group } \\
(n=15)\end{array}$ & $\begin{array}{c}\text { Matched controls } \\
(n=45)\end{array}$ \\
\hline Age (years) & $15.06 \pm 2.13$ & $14.66 \pm 2.00$ \\
Sex, males $(\%)$ & 40 & 40 \\
BMI & $24.04 \pm 3.91$ & $24.70 \pm 5.77$ \\
IGF1 $(\mathrm{ng} / \mathrm{ml})$ & $911.48 \pm 142.64$ & $915.26 \pm 191.75$ \\
IGFBP3 $(\mu \mathrm{g} / \mathrm{ml})$ & $2.62 \pm 0.32$ & $2.65 \pm 0.42$ \\
\hline
\end{tabular}

IGFBP3, IGF binding protein-3.

occasional family studies or identified common variants influencing stature via genome-wide association studies. This study differs from those in that we focused on a few specific genetic variations in IGF1R that plausibly attenuate IGF1 signaling and measured the effect in a normal adolescent population. We find that harboring a single copy of certain IGF $1 R$ variants leads to a substantial $(4 \mathrm{~cm})$ decrease in height on average, an observation supported by a recent computational analysis that predicts functional effects of these specific variants (22). This indicates that variation in height within healthy individuals may be explained, in some cases, by larger effects of a small subset of gene variants, rather than the combined small effects of many genes. Though the frequency of such IGF1R variants reported here is very low, we sought a highly restricted number of specific genetic variants. Thus, it is likely that additional rare variations of IGF $1 R$ also influence height within the normal population.

Our finding that these IGF1R variants were not associated with changes in circulating concentrations of IGF1 and IGFBP 3 is somewhat unexpected. If the IGF receptor changes we found result in a state of IGF resistance, then one would anticipate a reciprocal increase in GH secretion resulting in higher levels of IGF 1 and IGFBP 3 in the circulation. We reported this in the case with compound heterozygosity for IGF1R mutations (10). Because we made our measures in the postpubertal state, it is conceivable that there is a developmental or age-sensitive effect of the variants on circulating IGF1 concentrations that disappears postpuberty. However, increased concentrations of IGF1 and IGFBP3 have not been uniformly observed in other cases of biologically significant IGF1R mutations, including some with severe short stature $(6,9,15,16)$. The exact reasons for this are unclear but likely reflect the wide variation in serum levels among the general population and the multiple variables influencing circulating IGF1 concentrations.

We selected one of our genetic variants based on the family described by Inagaki et al. (7). Surprisingly, the individuals reported by Inagaki et al. were much more severely affected by the mutation than our cases, with heights from the former being in the -5 to -6 s.D. range. The authors verified that these mutations were biologically significant by demonstrating that cultured cells expressing the mutant IGF1R showed reduced
IGF1R signaling and proliferation in response to stimulation with IGF1. It is possible that the more severe phenotype reported by Inagaki et al. is explained by additional genetic variations that add to the effect of reduced IGF1 signaling. In support of the latter, there were modest reductions in stature $(-1.2$ to -2.2 s.D. below average) in the presumably non-affected family members.

The strengths of this study are that we selected IGF1R variants of proven or plausible biological significance and examined the effect in a representative population of normal adolescents with no other a priori assumptions as to results. A weakness of our study is lack of complete growth measurements, including birth anthropometrics, on all participants and the use of indirect measures to stage puberty. These shortcomings reflect the fact that the original purpose of the study was to examine metabolic state of the population, not growth and puberty. Thus, the height deficit we report could reflect modest growth attenuation throughout life or could be due to differences in the pubertal spurt (timing or magnitude). We could not assess the effects of the variants on prepubertal growth as they had all entered puberty at the time of enrollment. Nonetheless, the effect on stature was clearly evident with an appropriate statistical approach. Furthermore, the possibility that the variants simply influence pubertal timing and not final height is remote because the postpubertal state, as defined, placed the subjects very close to final height (e.g. $>2$ years post-menarche for females), measurements of circulating sex steroids were not lower in variants compared with controls, and analysis of consecutive measures of height indicated that growth had ceased in the variants and comparison group.

Another weakness is that we did not survey all known pathogenic IGF1R variants, including copy number variations. In some cases, this was because of technical limitations as noted previously; in others, the gene variants had not been reported at the time we conducted the study. However, the main purpose was not to determine the frequency and effect size of all known IGF1R variants but rather to test the hypothesis that rare IGF $1 R$ variants will produce relatively larger effects on stature in the general population. Indeed, this is what we found, providing further support for the critical role of the IGF1R in the pathway of growth control in humans.

\section{Declaration of interest}

S D Chernausek received research funding for this project from Eli Lilly and Company. A R Kansra, L M Dolan, L J Martin, and R Deka have nothing to disclose.

\section{Funding}

This work was supported by grants from Eli Lilly and Company, the National Institutes of Health (RO 1 DK59183), and an Institutional 
Clinical and Translational Science Award, NIH/NCRR (1UL1RR026314-01). Its contents are solely the responsibility of the authors and do not necessarily represent the official views of the NIH.

\section{Acknowledgements}

The authors wish to thank Walter Banach for his expertise in performing the hormone assays.

\section{References}

1 Perola M, Sammalisto S, Hiekkalinna T, Martin NG, Visscher PM, Montgomery GW, Benyamin B, Harris JR, Boomsma D, Willemsen $\mathrm{G}$ et al. Combined genome scans for body stature in 6,602 European twins: evidence for common Caucasian loci. PLoS Genetics 20073 e97. (doi:10.1371/journal.pgen.0030097)

2 Lango Allen H, Estrada K, Lettre G, Berndt SI, Weedon MN, Rivadeneira F, Willer CJ, Jackson AU, Vedantam S, Raychaudhuri S et al. Hundreds of variants clustered in genomic loci and biological pathways affect human height. Nature $2010 \quad 467$ 832-838. (doi:10.1038/nature09410)

3 Chan Y, Holmen OL, Dauber A, Vatten L, Havulinna AS, Skorpen F, Kvaloy K, Silander K, Nguyen TT, Willer C et al. Common variants show predicted polygenic effects on height in the tails of the distribution, except in extremely short individuals. PLoS Genetics 20117 e1002439. (doi:10.1371/journal.pgen.1002439)

4 Liu J-P, Baker J, Perkins AS, Robertson EJ \& Efstratiadis A. Mice carrying null mutations of the genes encoding insulin-like growth factor I (Igf-1) and the type 1 IGF receptor (Igf1r). Cell 199375 59-72.

5 Ester WA, van Duyvenvoorde HA, de Wit CC, Broekman AJ, Ruivenkamp CA, Govaerts LC, Wit JM, Hokken-Koelega AC \& Losekoot M. Two short children born small for gestational age with insulin-like growth factor 1 receptor haploinsufficiency illustrate the heterogeneity of its phenotype. Journal of Clinical Endocrinology and Metabolism $2009 \mathbf{9 4}$ 4717-4727. (doi:10.1210/jc. 2008-1502)

6 Fang P, Schwartz ID, Johnson BD, Derr MA, Roberts CT Jr, Hwa V \& Rosenfeld RG. Familial short stature caused by haploinsufficiency of the insulin-like growth factor i receptor due to nonsensemediated messenger ribonucleic acid decay. Journal of Clinical Endocrinology and Metabolism 200994 1740-1747. (doi:10.1210/jc.2008-1903)

7 Inagaki K, Tiulpakov A, Rubtsov P, Sverdlova P, Peterkova V, Yakar S, Terekhov S \& Leroith D. A familial IGF-1 receptor mutant leads to short stature: clinical and biochemical characterization. Journal of Clinical Endocrinology and Metabolism $2007 \mathbf{9 2}$ 1542-1548. (doi:10.1210/jc.2006-2354)

8 Walenkamp MJ, van der Kamp HJ, Pereira AM, Kant SG, van Duyvenvoorde HA, Kruithof MF, Breuning MH, Romijn JA Karperien M \& Wit JM. A variable degree of intrauterine and postnatal growth retardation in a family with a missense mutation in the insulin-like growth factor I receptor. Journal of Clinical Endocrinology and Metabolism 200691 3062-3070. (doi:10.1210/jc.2005-1597)

9 Kawashima Y, Kanzaki S, Yang F, Kinoshita T, Hanaki K, Nagaishi JI, Ohtsuka Y, Hisatome I, Ninomoya H, Nanba E et al. Mutation at cleavage site of IGF receptor in a short stature child born with intrauterine growth retardation. Journal of Clinical Endocrinology and Metabolism 200590 4679-4687. (doi:10. 1210/jc.2004-1947)

10 Abuzzahab MJ, Schneider A, Goddard A, Grigorescu F, Lautier C, Keller E, Kiess W, Klammt J, Kratzsch J, Osgood D et al. IGF-I receptor mutations resulting in intrauterine and postnatal growth retardation. New England Journal of Medicine 2003349 2211-2222. (doi:10.1056/NEJMoa010107)
11 Wallborn T, Wuller S, Klammt J, Kruis T, Kratzsch J, Schmidt G, Schlicke M, Muller E, van de Leur HS, Kiess W \& Pfaffle R. A heterozygous mutation of the insulin-like growth factor-I receptor causes retention of the nascent protein in the endoplasmic reticulum and results in intrauterine and postnatal growth retardation. Journal of Clinical Endocrinology and Metabolism 201095 2316-2324. (doi:10.1210/jc.2009-2404)

12 Kruis T, Klammt J, Galli-Tsinopoulou A, Wallborn T, Schlicke M, Muller E, Kratzsch J, Korner A, Odeh R, Kiess W \& Pfaffle R. Heterozygous mutation within a kinase-conserved motif of the insulin-like growth factor I receptor causes intrauterine and postnatal growth retardation. Journal of Clinical Endocrinology and Metabolism $2010 \quad 95$ 1137-1142. (doi:10.1210/jc. 2009-1433)

13 Mohn A, Marcovecchio ML, de Giorgis T, Pfaeffle R, Chiarelli F \& Kiess W. An insulin-like growth factor-I receptor defect associated with short stature and impaired carbohydrate homeostasis in an Italian pedigree. Hormone Research in Pediatrics $2011 \mathbf{7 6}$ 136-143. (doi:10.1159/000324957)

14 Fang P, Cho YH, Derr MA, Rosenfeld RG, Hwa V \& Cowell CT. Severe short stature caused by novel compound heterozygous mutations of the insulin-like growth factor 1 receptor (IGF1R). Journal of Clinical Endocrinology and Metabolism 201297 E243-E247. (doi:10.1210/jc.2011-2142)

15 Choi JH, Kang M, Kim GH, Hong M, Jin HY, Lee BH, Park JY, Lee SM, Seo EJ \& Yoo HW. Clinical and functional characteristics of a novel heterozygous mutation of the IGF1R gene and IGF1R haploinsufficiency due to terminal $15 \mathrm{q} 26.2 \rightarrow$ qter deletion in patients with intrauterine growth retardation and postnatal catch-up growth failure. Journal of Clinical Endocrinology and Metabolism 201196 E130-E134. (doi:10.1210/ jc. 2010-1789)

16 Veenma DC, Eussen HJ, Govaerts LC, de Kort SW, Odink RJ, Wouters $\mathrm{CH}$, Hokken-Koelega AC \& de Klein A. Phenotypegenotype correlation in a familial IGF1R microdeletion case. Journal of Medical Genetics 201047 492-498. (doi:10.1136/jmg. 2009.070730)

17 Kawashima Y, Higaki K, Fukushima T, Hakuno F, Nagaishi JI, Hanaki K, Nanba E, Takahashi SI \& Kanzaki S. Novel missense mutation in the IGF-I receptor L2 domain results in intrauterine and postnatal growth retardation. Clinical Endocrinology 201277 246-254. (doi:10.1111/j.1365-2265.2012.04357.x)

18 Dolan LM, Bean J, D'Alessio D, Cohen RM, Morrison JA, Goodman E \& Daniels SR. Frequency of abnormal carbohydrate metabolism and diabetes in a population-based screening of adolescents. Journal of Pediatrics 2005146 751-758. (doi:10.1016/j.jpeds.2005.01.045)

19 Obesity and cardiovascular disease risk factors in black and white girls: the NHLBI Growth and Health Study. American Journal of Public Health 199282 1613-1620. (doi:10.2105/AJPH. 82.12.1613)

20 Morrison JA, Sprecher DL, Biro FM, Hansen CA, Lucky AW \& Wride K. Sex hormones and lipoproteins in adolescent male offspring of parents with premature coronary heart disease and a control group. Journal of Pediatrics 1998133 526-532. (doi:10.1016/S0022-3476(98)70062-9)

21 Singer JD. Using SAS PROC MIXED to fit multilevel models, hierarchical models, and individual growth models. Journal of Educational and Behavioral Statistics $1998 \quad 24$ 323-355. (doi:10.3102/10769986023004323)

22 de Alencar SA \& Lopes JC. A comprehensive in silico analysis of the functional and structural impact of SNPs in the IGF1R gene. Journal of Biomedicine \& Biotechnology 20102010715139. (doi:10.1155/2010/715139)

Received 28 February 2012

Revised version received 28 August 2012

Accepted 12 September 2012 